\title{
Identifying Impostors in Architectural Education
}

\author{
SARAH YOUNG \\ University of Louisiana at Lafayette
}

The impostor phenomenon (IP) is a feeling of incompetence despite evidence of competence. In addition to feelings of intellectual phoniness, impostor feelings are often accompanied by anxiety, depression, and psychological distress. Impostor feelings arise most frequently when encountering new challenges and when feeling like an outsider within your peer group or discipline; as such, IP has been well-documented in college students across many disciplines. IP has yet to be studied in the context of architecture education, where unique additional challenges may exacerbate impostor feelings; challenges confronted during the design process, frequent and public critiques and reviews, the competitive and comparative atmosphere, the overwhelming array of skills and knowledge to acquire, and demanding workloads may contribute to feelings of incompetence, even if evidence of competence exists. If architecture students suffer from IP, it is imperative that these issues be addressed as we strive to make the academy and the profession more humane and inclusive. The design studio experience is for learning how to design as both a creative process AND a healthy, sustainable practice - in academic and future professional life.

The purpose of this study was to discover whether first year design students identify with impostor characteristics, and if so, whether their IP feelings grow or diminish over the course of the semester. The Clance Imposter Phenomenon Scale (CIPS) was used to study the incidence of IP in first semester design studio. Most students surveyed were found to experience either "moderate," "frequent," or "intense" impostor feelings. The paper presents these findings and explores methods of lessening the impact of IP in the design studio through dialogue, building trust, reaching out to social networks from outside the studio, giving feedback which helps students attribute success to their abilities, and helping students learn to self-validate.

\section{THE IMPOSTOR PHENOMENON}

The imposter phenomenon (IP) was first identified by clinical psychologists Clance and Imes in a 1978 study of highachieving women, many of whom felt a sense of intellectual phoniness. The study found that those experiencing IP believe that they are "really not bright and have fooled anyone who thinks otherwise and often attribute their success to external factors, even in the presence of evidence to refute this belief ${ }^{\prime \prime}{ }^{1}$
Parkman describes the impostor phenomenon as an "inability to accurately self-assess with regard to performance" which leads sufferers to deal with the resulting anxiety and stress by "working longer, working harder and seeking perfection." Though IP relates to both depression and social anxiety, has been distinguished as a separate construct. ${ }^{3}$

"I feel so emotionally drained, without any confidence in my ability."

-Anonymous Student Respondant, Architects' Journal's 2016 Annual Student Survey

In 2016, Parkman's comprehensive review of studies of IP in higher education found the phenomenon to be pervasive in university students, staff, and faculty as well as detrimental to both individual and organizational health. Parkman asserts that, "there is enough documentation to support the integration of programming on campus and reflection upon how the academy feeds [the impostor phenomenon]." In college students, IP has been found to positively correlate with anxiety, depression, psychological distress, and minority student status stress. ${ }^{5}$

While research shows that college campuses feed the impostor phenomenon, there has yet to be a study of IP in architecture education. Considering the growing body of research on the detrimental effects of architectural education on student mental health, IP in this context deserves examination.

\section{IP + ARCHITECTURE EDUCATION}

Since Clance and Imes' initial study on high achieving women, IP has been studied and identified in a variety of settings across demographic groups. Evidence now suggests that sex is not a determining factor of IP, however, IP has been found to occur more frequently in those who are racial and/or gender minorities in their disciplines. ${ }^{6} \mathrm{IP}$ also appears to be more prevalent in individuals who are encountering new challenges. ${ }^{7}$ This makes the issue of IP particularly relevant to college students. Emerging adults experiencing IP are "caught in a cycle in which they require external sources of validation (e.g., praise, comparisons to the performance of others) to feel competent, and yet, when they receive such validation, they engage in discrediting behaviors that prevent them from internalizing 
CIPS Test Results in a First Semester First Year Design Studio: WEEK 3
\# of Participants $=36$

Mean Score $=62.14$

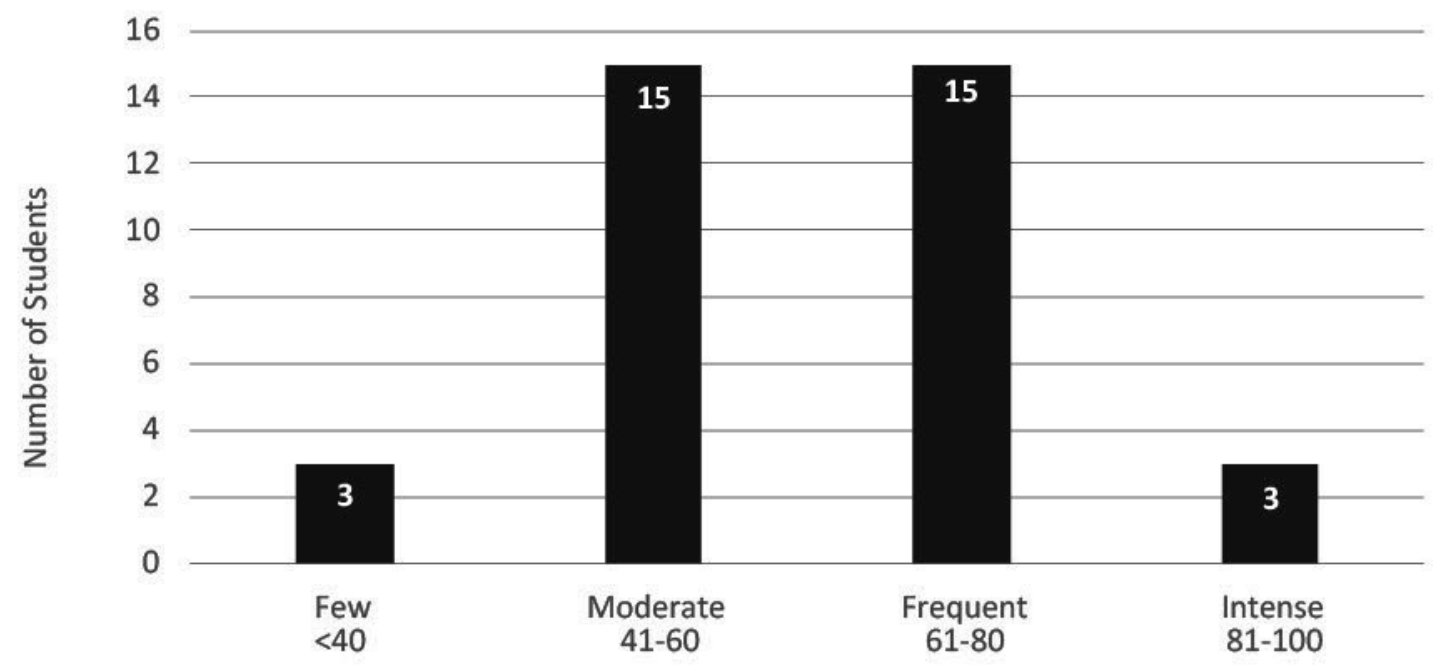

Frequency of Impostor Phenomenon Experiences / Overall CIPS Scores

Figure 1: CIPS Test Results in a First Year First Semester Design Studio, Week 3, Copyright 2019, Sarah Young.

it." ${ }^{\prime \prime}$ IP characteristics have been found to be prevalent among undergraduate, graduate, and PhD students alike, and often most intensely affects high-achieving students, students whose parents emphasize high achievement, ${ }^{9}$ first-generation college students, ${ }^{10}$ students enrolled in highly selective universities, ${ }^{11}$ and students who are minorities of some kind in their disciplines or in the university at large. ${ }^{12}$

While the transition to university life is challenging for all college students, the educational environment of the architecture studio can present unique additional challenges which could exacerbate impostor feelings. Frequent and public critiques and reviews, an overwhelming array of skills and knowledge to acquire, demanding workloads, and cliquish studio cohorts all contribute to a competitive, comparative atmosphere in the design studio. The design process itself presents challenges; much knowledge required to create architecture is experiencebased and remains tacit, creating a steep and often frustrating learning curve. Risking failure in front of peers and professors can be uncomfortable for most and excruciating for others.

These conditions may lead many students to frequently question their fitness to pursue a degree in architecture. While racial and gender diversity is improving in architectural schools, attrition rates remain low, indicating a continued need for improved support. Furthermore, the commonality of high rates of stress, anxiety, and depression among architecture students is deeply disturbing and cannot be ignored. Impostor feelings can cloud objective critical thinking, foster self-doubt and unhealthy work practices ("sleep is for the weak") and exacerbate mental health issues. Semester after semester as the stakes get higher, the mental and physical health impacts can become increasingly crushing and dire. ${ }^{13}$ Left unaddressed, these habits of work and mind follow students into the profession.

In design studios, the "symptoms" of IP are anecdotally and statistically evident. If architecture students are found to suffer from the impostor phenomenon, architecture educators should be armed with the tools to respond.

\section{TESTING FOR IP IN A DESIGN STUDIO}

The purpose of the study was to discover whether first year design students identify with impostor characteristics, and if they do, whether IP feelings grow or diminish over the course of the semester. Test participants were design students in a first semester design studio class for architecture, interior design, and industrial design majors. In this design foundations course, projects are not discipline specific and the emphasis is on learning how to design through a process of iteration.

First semester design students were asked to complete the Clance Impostor Phenomenon Scale (CIPS) ${ }^{14}$ test, a 20-item survey developed by a clinical psychologist to measure the frequency and intensity of impostor feelings in individuals. For each item, participants select how strongly they identify with a statement relating to impostor feelings on a Likert-type scale, ranging from 1 (not at all true) to 5 (very true.) The sum 
CIPS Test Results in a First Semester First Year Design Studio: WEEK 11

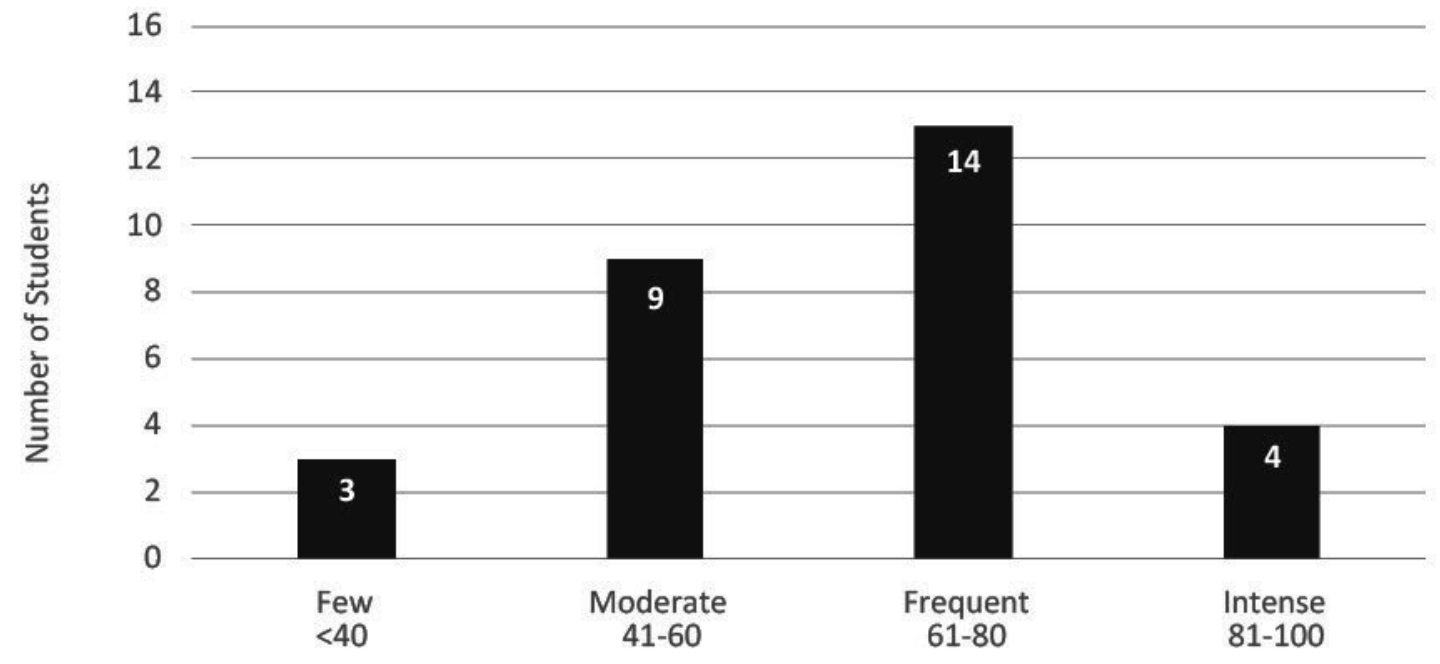

Frequency of Impostor Phenomenon Experiences / Overall CIPS Scores

Figure 2: CIPS Test Results in a First Year First Semester Design Studio, Week 3, Copyright 2019, Sarah Young.

of these scores yields a total score; the higher a person scores, the more frequently and intensely IP experiences interfere with their life. The CIPS tests for 3 overall dimensions of IP: concern about intelligence and ability, feelings that accomplishments are due to chance or error, and the inability to accept good performance or acknowledge praise. ${ }^{15}$

Participation in the study was voluntary and anonymous to protect students' privacy. Since the study was being conducted by their teacher, the teacher left the room during the 15-minute survey period to protect students' anonymity and remove pressure to unwillingly participate. Students could choose to withdraw from the study and refrain from answering any questions for any time and for any reason. At the time this paper was written, the CIPS test had been given twice, with the intention of being given three times over the course of a semester. The first test was administered during the third week of class, the second during the eleventh week, and the third will be given during the final week of the semester.

\section{WEEK 3 CIPS TEST RESULTS}

36 out of 50 students enrolled in the course ( $72 \%$ of the class) participated in the first CIPS test in Week 3 of the semester. Results showed that many students surveyed identified with impostor characteristics to some degree, with $91.6 \%$ of students reporting moderate to intense IP feelings. 8.3\% of students received a score of 40 or less (few IP characteristics.) $41.6 \%$ of students scored between 41-60 (moderate
IP characteristics.) $41.6 \%$ of students scored between $61-80$ (frequent IP characteristics.) $8.3 \%$ of students received a score of 81-100 (intense IP characteristics.) As first year college students, these results could be due in part to the participants adjusting to life in college, or they could be indicative of anxiety created by the new environment of the design studio. Regardless of the cause, the results show that most students surveyed feel like impostors sometimes, and exactly $50 \%$ felt like impostors either frequently or intensely. Of the 20 test items, those which received the highest average scores were items related to concern about intelligence and ability.

\section{WEEK 11 CIPS TEST RESULTS}

The second test, conducted in Week 11 of the semester, had fewer student participants, with 29 of 43 enrolled students participating ( $67 \%$ of the class). There are a few factors which could explain the slightly lowered level of participation. Some students withdrew from the class between the first and second CIPS tests. Others chose to continue working on their projects instead of participating in the study that day. Others may not have been interested in continuing to participate for personal reasons. The results showed that of students surveyed, $89.7 \%$ experienced moderate to intense IP characteristics. $10.3 \%$ of students scored 40 or less (few IP feelings.) $31.0 \%$ of students scored between $41-60$ (moderate IP feelings.) $44.8 \%$ of students scored between $61-80$ (frequent IP feelings.) $13.7 \%$ of students received a score of 81-100 (intense IP feelings.) 
Superficially, the results could indicate an increase in impostor characteristics between the first test in Week 3 of the semester and second test in Week 11. Due to the voluntary and anonymous nature of the test and slightly diminished participation in an already small sample, results are inconclusive as to whether impostor feelings have grown, diminished, or remained constant over the course of the semester. However, results are clear that many of design students surveyed continued to identify strongly with impostor characteristics. As in the first test, the items which received the highest average scores were those related to concern about intelligence and ability. This finding suggests the use of altered tactics in the design studio to combat these feelings.

\section{TACKLING IP IN THE DESIGN STUDIO}

The following are strategies for tackling IP in the design studio gleaned through research and discussion during the conference presentation. Many of the strategies discussed are concerned with "soft skills" in design and design teaching. Through these means, a studio culture that is productive, creative, and rigorous as well as healthy, reflective, and supportive can be cultivated.

Starting a Dialogue: Talk IS action. One effective means of learning to cope with IP is to hear peers and mentors discuss it from their own experience. Research shows that learning about IP through taking a survey like the CIPS can be relieving in itself. ${ }^{16}$ Alongside simple awareness of IP, in architecture education it is important to create a culture where students feel they can talk about failure and mistakes, as this makes students who feel like impostors more likely to reach out for help when they need it. ${ }^{17}$ This means encouraging students discuss the difficulties they are encountering, not in a support program, but at the site where the angst occurs - in the studio. By breaking taboo and discussing IP in class, otherwise isolated students can be empowered to seek support. Furthermore, conversations in which participants can be vulnerable build trust.

Building Trust: Acquisition of tacit design knowledge requires learners to take risks and leaps of faith that require trust: trust in the educator, trust in the system, and trust in the process. By engaging in discussions about failure and mistakes as a participant rather than moderator, trust can be built between teachers and students. Knowing that mentors and figures they look up to also struggle with impostor feelings can help students feel less isolated and provide needed perspective. ${ }^{18}$ For students to trust the system of the design studio, transparency should be promoted and promises kept. Sticking to set deadlines, developing grading criteria that can be easily understood by students, and providing feedback which relates to those criteria can give students a foothold on a path that may seem treacherous at first. Finally, for students to trust the process enough to take the leaps of faith involved in acquiring tacit design knowledge, educators must attempt to understand the project a student is trying to achieve to the same degree as a student is trying to understand what is expected of them.
Bolstering Social Support: A recent study found that students who struggled with IP often felt worse if they "reached in" to other students within their major" for social support. ${ }^{19}$ Conversely, students felt their impostor feelings reduce if they "reached out" to support systems outside of their classrooms. Reaching outside of the discipline for support allows students to "understand themselves more holistically rather than being so focused on what they felt they lacked in just one area." ${ }^{20}$ Furthermore, architecture students frequently rely on each other ("the only ones who understand") for emotional support, which can place an unintentional burden on classmates who have enough to manage on their own. Rather than create a situation where students remain in isolation for much of the semester, giving students a break and explicitly encouraging them to catch up with friends and family could go a long way towards helping students cope, both with IP and the pressures of studio in general. Additionally, while a great deal of bonding takes place in studio cohorts, studios can be cliquish and leave some individuals on the outside. On a larger scale, studio cohorts and architecture programs often become larger cliques within the university. On one hand, this can lead to a strong sense of community, but on the other it can create isolated microcosms in which architecture students feel apart from other university students. This can make architecture students less likely to take advantage of health and wellness or social programming designed for the student body at large. One way to mediate this in the design studio is to seek opportunities to involve students in interdisciplinary or community-based projects in which they can share their unique expertise. Interacting with others who have specialized knowledge can help students validate their own knowledge as well as develop social networks outside of the architecture studio.

Aiding Self-Attribution: A common feeling among IP sufferers is that their success can be attributed either to luck or to the help they have received. These feelings can be combated through feedback that helps students attribute success to their acquired knowledge and abilities. During a critique it is important to validate that a design project is not only good, but that the student has learned something to make it so. This acknowledgement makes a student's tacit, personal knowledge explicit and can bolster self-confidence in their abilities. Another issue related to self-attribution is that students who feel like impostors may believe that their project is only good because of the feedback they received from their teacher. In our discussion at this year's conference, a presentation attendee addressed this confusion of ownership, saying that he often tells students that, "advice is given to you, if you take it, it's yours." This makes the transfer of knowledge from educator to student clearer to the student. It is important to remember that transfer is authetic only when a student is truly free to take or leave the advice. 
Fostering Self-Validation: Another common difficulty among IP sufferers is the inability to accurately assess their performance. This causes those with impostorism to rely heavily on external validation of their work to feel competent. For a student to become an independent designer, they must learn to assess their own work instead of seeking a professor's critique at every step. A practice of metacognitive work can help students learn critical skills necessary for self-validation. ${ }^{21}$ Metacognitive assignments focus students' attention on 3 critical factors: evaluating past work (e.g. "How do I know which exploratory model is most successful?"), monitoring current progress (e.g. "How can I gauge whether I'm on the right track?"), and planning next steps. (e.g. "How can I set myself up to have a productive work session this Saturday?") ${ }^{22}$ Assignments which promote self-evaluating, self-monitoring, and planning ahead can build habits of mind that promote independence. Keeping a metacognitive journal allows students to make tacit knowledge not just explicit, but external, and available to revisit at a later date. Additionally, spending time in the studio to reflect on the semester overall can help students consider which work habits served them well in the past and which ones they would like to avoid in the future.

\section{A CALL TO ACTION}

While many architecture and design students may suffer from the impostor phenomenon, they do not have to suffer alone. There are several strategies for making a positive impact; initiating a dialogue, building trust, reaching out to social networks from outside the studio, giving feedback which helps students attribute success to their abilities, and helping students learn to self-validate can help create a healthier studio culture in which individuals and the community can thrive. The design studio experience is for learning how to design as both a creative process AND a healthy, sustainable practice - in academic and future professional life. As we strive to make the academy and the profession more humane and inclusive, it is imperative that IP issues be addressed early in architecture students' education, before unhealthy habits of work and mind take hold. Discussing the impostor phenomenon early and often can help students understand they are not impostors: they are beginners.

\section{ENDNOTES}

1. Clance, Pauline R, and Suzanne Ives. "The impostor phenomenon in high achieving women: Dynamics and therapeutic intervention," Psychotherapy: Theory, Research, and Practice 15, no. 2 (Fall 1978): 241-247.

2. Parkman, Anna. "The Imposter Phenomenon in Higher Education: Incidence and Impact," Journal of Higher Education Theory and Practice 16, no. 1 (2016): 52.

3. Chrisman Sabine M, W. A. Pieper, Pauline R Clance, C.L. Holland, and Cheryl Glickauf-Hughes. "Validation of the Clance Impostor Phenomenon Scale," Journal of Personality Assessment 65, no. 3 (1995): 457.

4. Parkman, 52.

5. Ibid, 55-56.

6. Ibid, 56 .

7. Weir, Kirsten. "Feel Like a Fraud?" American Psychological Association https://www.apa.org/gradpsych/2013/11/fraud (retrieved November 7, 2019).
8. Lane Joel A. "The Imposter Phenomenon Among Emerging Adults Transitioning Into Professional Life: Developing a Grounded Theory," Adultspan Journal 14, no. 2 (October 2014): 115.

9. King, Julie E, and Eileen Cooley. "Achievement Orientation and the Impostor Phenomenon among College Students," Contemporary Educational Psychology 20, no. 3 (July 1995).

10. Stebleton, Michael, and Krista Soria. "Breaking down barriers: Academic obstacles of first-generation students at research universities," The Learning Assistance Review 17, no. 2 (2012): 15

11. Woodley, Lucas Y. "Acceptance To Harvard, Impostor Syndrome, and What It All Means," The Harvard Crimson. https://www. thecrimson. com/article/2019/9/5/woodley-acceptance-to-harvard/ (retrieved November 12, 2019)

12. Cokley, Kevin, Shannon McClain, Alicia Enciso, and Mercedes Martinez. "An Examination of the Impact of Minority Status Stress and Impostor Feelings on the Mental Health of Diverse Ethnic Minority College Students," Journal of Multicultural Counseling and Development 41, no. 2 (April 2013)

13. Block, India. "Architecture students "considered suicide" over long hours, say \#dezeenchat participants," Dezeen. https://www.dezeen.com/2019/07/16/ burn-out-design-education-mental-health/ (retrieved August 5, 2019).

14. Clance Impostor Phenomenon Scale (CIPS). From The Impostor Phenomenon: When Success Makes You Feel Like A Fake (pp. 20-22), by P.R. Clance, 1985, Toronto: Bantam Books. Copyright 1985 by Pauline Rose Clance, Ph.D., ABPP. Use by permission of Dr.Pauline Rose Clance. Do not reproduce/copy/distribute without permission from Pauline Rose Clance, drpaulinerose@comcast.net, www.paulineroseclance.com.

15. Chrisman, 465

16. Lane, 122.

17. Gardner, Richard G, Jeffery S Bednar, Bryan W Stewart, James B Oldroyd, and Joseph Moore "“I must have slipped through the cracks somehow": An examination of coping with perceived impostorism and the role of social support," nation of coping with perceived impostorism
Journal of Vocational Behavior 115 (2019).

18. Dancy II, T Elon, and M Christopher Brown. "The Mentoring and Induction of Educators of Color: Addressing the Impostor Syndrome in Academe," Journal of School Leadership 21, no. 4 (2011)

19. Gardner

20. Ibid.

21. McCombs, Barbara L."The role of the self-system in self-regulated learning," Contemporary Educational Psychology 11, no. 4 (October 1986).

22. Young, Sarah. "Write from the Start: Using Writing to Enhance the Design Process in a First-Year Design Studio," in 2018 Design Communications Association Conference Proceedings. ed. M Saleh Uddin, So-Yeon Yoon, Lora Kim, Christopher Welty, (2018), 24-30. 